\title{
Virtual Reality-Based Skills Transfer and Human Resource Development Based on Brain Activity Assessment
}

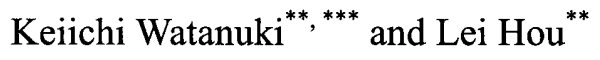 \\ **Graduate School of Science and Engineering, Saitama University, \\ ${ }^{\star * \star}$ Saitama University Brain Science Institute, \\ 255 Shimo-okubo, Sakura-ku, Saitama-shi, Saitama 338-8570, JAPAN \\ E-mail: watanuki@mech.saitama-u.ac.jp
}

\begin{abstract}
This paper proposes a new virtual reality-based job training and human resource development. In our proposed system, the education content is displayed in the immersive virtual environment with haptic information, whereby a trainee may experience work in the virtual site operation. The brain functional activities of the worker under virtual and real skills training are measured using near-infrared spectroscopy, and the characteristics of these activities are analyzed in detail.
\end{abstract}

Key words: Brain activity, Near-infrared spectroscopy, Virtual reality, Lathe operation

\section{Introduction}

Despite a general trend toward automated lathe operation with NC lathes and other systems, many types of high-precision machining are difficult to automate and require manual lathe operation by highly skilled machinists with years of experience who can judge the state of the machining operation by sensing chatter vibration, sounds, and even smells generated during lathe turning. This ability is generally referred to as "instinct", or simply "skill". In recent years, however, with the widespread aging of skilled lathe operators, concern is rising that this skill could be lost [1]. In the past, it was learned at the side of a master machinist, who guided the trainee in the actual use of the lathes. Instruction was given chiefly through physical guidance, rather than by verbal explanation. In this on-the-job training (OJT), the operating skill was thus learned in guided experience and the trainee was in this way able to gain the required implicit knowledge, awareness, and sensitivity to potential problems in lathe operation [2]. This learning method, however, is time- and labor-intensive, and it is highly dependent on the capabilities of the trainer in nurturing the requisite skill.

The authors have proposed a training method which merges OJT with a virtual reality (VR) environment for physical experience in the processing operation [3]. In this method, lathe operation can be readily repeated under given conditions. It also has the advantage of providing safe, virtual training and experience in the occurrence of accidents and their consequences due to errors in operation. In this paper, we describe the construction of a VR system for the transmission of lathe operation skill, and neurological analysis of the effect on the trainee of using the VR system as compared with training on an actual general-purpose lathe, based on near-infrared spectroscopy (NIRS) measurements of brain activity during lathe operation with the VR system and with an actual lathe. 


\section{Methods of skills transfer}

\subsection{Present Situation and Problem Points}

As the pace of technical change becomes faster and competition between companies becomes more severe, it is necessary for each company to try to learn faster than the other. Therefore, the number of companies undertaking knowledge management, such as knowledge sharing based on organizational learning theory, knowledge transfer, etc., is increasing, advanced technologies are being recorded in manuals, and information databases using ICT are being introduced. However, as the division of labor in manufacturing sites increases, obstacles to information sharing arise, such as the attitude "as long as it follows the manual, this is accurate enough for my work," and not much thought is given even though accidents occur or defective products are produced. Therefore, there is a danger that knowledge management, which aims to share knowledge and skills among members of an organization in order to improve the rate of learning, can have the opposite effect and actually hinder the nurturing of skilled operatives. Also, if too much reliance is placed on knowledge accumulated in databases within the company, the opportunities for problem solving on one's own reduce. Therefore, by making knowledge sharing easier in order to quickly develop human resources capable of raising efficiency, learning by experience is hindered, so when problems occur it is not possible to quickly respond to them. This leads to the dilemma that knowledge sharing can result in higher costs.

A skilled worker is one that within a specific field has both specialist training and practical experience, has acquired special skills and knowledge, has outstanding "structured knowledge" in that specific field based on their experience and training, understands problems deeply, can accurately and quickly solve problems, and has excellent self-monitoring skills. To develop such a skilled worker, practical on-the-job training (OJT) and so on is carried out, but various problems with this system are evident. Examples include the fact that learning through failure is difficult structurally, there are time restrictions, depending on the circumstances appropriate guidance is not necessarily provided, individuals become satisfied with their level of learning at a certain stage, and thus the knowledge at the site becomes fixed, hindering adaptation to a new environment, etc.

Also, although the necessity of knowledge transfer by engineers and skilled operatives in the core manufacturing technology industries has already been pointed out, the sense of crisis in qualitative and quantitative shortages was strengthened with the "year 2007 problem." From 2007, the baby boomer generation, which was responsible for industry during the era of high growth rate in Japan, started to retire in large numbers, raising many concerns. A number of initiatives have been tried to solve the year 2007 problem, but there have been many drawbacks, for example, the skills transfer and know-how took time and did not proceed smoothly; the human resources systems providing guidance were insufficient; and other issues. The main background to these problems is that during the period of high economic growth (the period from roughly 1955 to 1975) new employees were employed in large numbers. Technology and skills were passed down through in-company training. However, after the oil-shock of 1973, energy efficiency, automation, and new technologies were rapidly introduced, and the traditional transfer of knowledge and skills tended to be neglected, which meant that the passing on of technology and skills to the younger generation slowed down. Also, from the economics viewpoint, due to the technology transfer associated with the relocation of factories abroad, mainly to South-East Asia, and with outsourcing, the hollowing-out of technology and skills within Japan has become serious. Following the collapse of the bubble economy in 1991, companies within Japan restructured, and the resultant cutbacks in capable technical and skilled staff, made the transferring of technology and skills even more difficult. Furthermore, as a result of the 
decline in the birthrate and the dislike for hard, dirty, or dangerous workplaces, it has become difficult to secure young engineers and skilled operatives. This has resulted in a large age gap between the baby boomer generation and the younger generation, so technology and skills are not smoothly passed down. As a result of this situation, measures to pass on technology and skills came into full swing.

At present, transferring skill is typified by technical documents, video libraries, and OJT. Creating technical documents and video libraries requires a lot of labor and cost, and despite their many merits they are not widely used due to poor ease of use. At present, most factories concentrate on OJT for transferring skills.

The problem points with conventional transfer skill methods are as follows. Technical documents are ideal for describing technologies, but they have the disadvantage that it is not possible to describe the movements and skills of operatives very well. Also, video libraries are good at recording and preserving skills, but a lot depends on the knowledge and capability of the person watching the video. Also, in the normal video format it is difficult to immediately watch the part you want to see. In contrast, OJT is an extremely good method of skills transfer, as the whole body can experience the training using a person's five senses of seeing, hearing, taste, smell, and touch. However, this requires much time, and there are problem points; for example, the opportunities for experience are limited by the production of many products in small quantities; and it also very much depends on the educational ability of the person being instructed.

In this way, each of the methods of skills transfer has many advantages, but also has a limit to transfer skills. In addition, looking at these methods from the viewpoint of knowledge conversion, with OJT and video libraries tacit knowledge is transmitted as tacit knowledge, but with technical documents tacit knowledge is converted into explicit knowledge for transmission. Here, explicit knowledge can be defined as "knowledge that can be represented in the language of documents and diagrams, etc." and "knowledge based on objective general laws," and so-called "technology" corresponds to explicit knowledge. On the other hand, tacit knowledge can be defined as "knowledge that is difficult to represent clearly in the language of documents and diagrams, etc." or "knowledge based on subjective or individual experience," and so-called "skills" correspond to tacit knowledge. Using these methods on their own, where tacit knowledge is transmitted as tacit knowledge or tacit knowledge is converted into explicit knowledge by omitting a part of the tacit knowledge, problems are experienced with the quality, quantity, or efficiency of the transmitted knowledge. In other words, with the main conventional methods of passing down skills, namely technical documents, video libraries, and OJT, effective skills transfer is not possible at present.

\subsection{New system for skills transfer}

In the casting process, besides explicit knowledge of the technology for making castings, tacit knowledge in the form of skills is necessary. In order to design and manufacture high-added-value products, it is necessary to obtain knowledge that skillfully combines this explicit knowledge and tacit knowledge, as well as master technologies and skills through OJT or the like.

In our study, we consider an example of the utilization of a skills transfer system and an immersive virtual environment system and their correspondence with the knowledge conversion processes of the SECI model. Here, (1) socialization is the process in which a user clarifies awareness of problems and becomes aware of tacit knowledge through OJT and work on site; (2) externalization is the process in which the user acquires explicit knowledge from documents, technical data, etc., using the skills transfer system; (3) combination is the process in which the user acquires knowledge from images, etc., presented in the form of a combination of explicit knowledge and tacit knowledge; and (4) 
internalization is the process in which the user makes the actions and learning their own, based on the knowledge obtained from the skills transfer system. When making knowledge one's own while using the skills transfer system in the externalization and combination processes and using the immersion type virtual shared environment system in the internalization process, acquisition of new tacit knowledge as well as the creation of new knowledge is possible.

The authors have developed a skills transfer system for efficient and effective knowledge acquisition using multimedia and VR technology in each of the knowledge conversion processes of the SECI model. This is an example where the utilization of the skills transfer system and the system using the VR technology correspond to the knowledge conversion processes of the model. Here, externalization may be substituted for the process in which a user obtains explicit knowledge from documents and technical data, etc., using a multimedia skills transfer system; combination may be substituted for the process in which the user acquires knowledge presented in the form of a combination of explicit knowledge and tacit knowledge; and internalization may be substituted for the process in which the user makes the acquired skills their own by mock experience within the VR space, based on the knowledge obtained from the skills transfer system. It is when making knowledge one's own while using the skills transfer system in the externalization and combination processes, and using the VR space in the internalization process, that the creation of new knowledge is possible.

\section{Measurement of brain activation response by NIRS}

\subsection{Brain optical imaging system}

During neural activity in the brain, humans transmit and process information and decide upon actions or responses. When neural activity occurs, blood flow and blood quantity increase in the tissue near the active neurons, and the ratio between oxygenated and deoxygenated hemoglobin in the blood changes. Hemoglobin characteristically changes in near-infrared (700 900 nm) absorbance in accordance with its oxygen content, and it is therefore possible to determine changes in the oxygen level in the hemoglobin by measurement of this absorbance by NIRS. In NIRS, the cranial target area of the subject is overlaid with a special holder for optical fibers which are inserted into the holder at selected measurement positions on the scalp, thus enabling non-invasive measurement of NIR incidence and reception. This configuration places little or no restriction on bodily movement of the subject, and the measurements can therefore be made with the subject in a natural state of posture and movement. In the present study, we thus used NIRS to determine the state of the hemoglobin oxygenation at the cerebral surface and on that basis performed real-time color mapping of the state of brain activity (the brain activation response) in the target regions.

\subsection{Measurement of motor cortex response using NIRS}

Before attempting measurements for the complex actions performed in lathe processing, we performed preliminary trial measurements of brain activation responses for the three basic actions of moving the center of gravity, bending at the waist, and rotating a handle. For each experiment, the subjects were two males in their twenties. The NIRS instrument used for the measurements was the Shimadzu OMM-3000. The measured positions were in region of the motor cortex only, as shown in Figure 1.

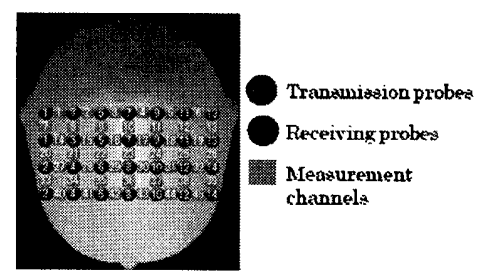

Fig.1 Regions of NIRS measurement

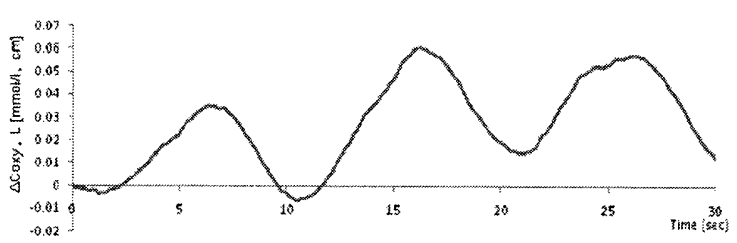

Fig.2 Brain activation response during waist bending 


\subsubsection{Waist bending trials}

In the waist bending trials, the subject repeatedly moved to a waist bending position after remaining in a standing position for $30 \mathrm{sec}$, and the brain activation response in the motor cortex was measured each time by NIRS. Each trial was performed twice. No instruction was given on the timing of the waist bending or the number of waist bends. Figure 2 shows an example of the relation found between the change in oxygenated hemoglobin concentration (oxyHb), as measured by NIRS, and the movement of the subject. In the motor cortex regions related to body trunk movement, oxyHb rose and fell three times as the subject performed waist bending three times, thus indicating that it is possible to determine the brain activation response relating to the waist bending movement by NIRS measurements. Further comparison between the subject's movement and the change in his trunk oxyHb shows that the oxyHb rose when waist bending was performed and tended to remain constant when the subject was stationary in a bent-waist posture. During the return to a standing position the oxyHb declined, and the decline ended when the subject next assumed the standing position. The results indicate that the degree of change in oxyHb in the motor cortex of the parietal region is highly dependent on the waist bending movement. It should also be noted that, as shown in Figure 2, the oxyHb changes were delayed by approximately $2 \mathrm{sec}$ from the time of the actual movement. This delay in oxyHb change was observed in all of the measurements of the present study, though some differences were observed among individual subjects, and it was thus inferred that the brain activation response as measured by NIRS is generally several seconds behind the actual bodily movement.

\subsubsection{Center of gravity movement trials}

In the trials relating to center of gravity movement, the brain activation response in the motor cortex was measured by NIRS while the su bject performed movement of his center of gravity for $30 \mathrm{sec}$. The position of the center of gravity during the movement was also simultaneously measured with a ground reaction meter. In a single trial, the subject moved his center of gravity in arbitrary directions once, to the left and right once, and fore and aft o nce, for a total of three movement segments. In this trial, no instruction whatsoever was given as to the timing of the movement, but the subject was instructed not to bend at the waist. Figure 3 shows the results for the center of gravity movement in arbitrary directions. The ground reaction meter measurements showed that the subject moved his center of gravity to the left during the period 0 to $4 \mathrm{sec}$. During the movement, the oxyHb change in the right hemisphere increased, and reached a maximum when the movement stopped. At 4 to $10 \mathrm{sec}$, during which the subject moved his center of gravity to the right, the oxyHb of the motor cortex in the left hemisphere increased and reached a maximum when the subject became stationary. In contrast, the oxyHb in the right hemisphere of the motor cortex declined during the movement and reached a minimum when the subject became stationary. The same trends were observed in the time segments of center of gravity movement to the left and right only. During the period 10 to $15 \mathrm{sec}$, the subject moved his center of gravity forward, and the oxyHb of the motor cortex declined in both the left and the right hemisphere, and at $14.13 \mathrm{sec}$ together reached a minimum in both hemispheres. During the period 15 to $20 \mathrm{sec}$, the subject was moving his center of gravity backward. If the trend was the same as that described above for the forward movement segment, during the backward movement the oxyHb of the motor cortex should have risen in both hemispheres until reaching a maximum in both, but in this segment of the trial the oxyHb in the motor cortex instead increased more rapidly in the right hemisphere than in the left and reached its maximum value there. The ground reaction meter results showed that, during the 10 to 15 sec segment, in which the subject performed forward movement of the center of gravity, the 
direction of the movement was essentially directly forward, but during the 15 to $20 \mathrm{sec}$ segment the subject performed not only backward movement of the center of gravity but also moved it towards the left. In the trial segment in which the subject moved his center of gravity left and right only, the oxyHb rose in the motor cortex in the direction of movement of the center of gravity. This suggests that in the trial segment in which the subject was to move the center of gravity backward, the center of gravity was actually tilted toward the left, thus resulting in a more rapid rise in the oxyHb in the right hemisphere of the motor cortex than in the left, and it reaching its maximum value in the former hemisphere. The results of the center of gravity trials thus indicate that it is possible to measure the brain activation response relating to the direction of the center of gravity movement using NIRS.

\subsubsection{Handle rotation trials}

In the trials involving crank handle rotation, the NIRS measurements were performed while the subject rotated the handle under several different loads. The handle was of the same configuration as those actually used in lathes. The handle torque was $0.009,0.025$, 0.651 , or $1.332 \mathrm{Nm}$. At the time of the trial, the subject was instructed to rotate the handle using the left and right hands by turns, each for $30 \mathrm{sec}$. The direction of rotation was counterclockwise, and no instruction was given as to the speed of the rotation. Figure 4 shows the oxyHb change in Subject A as he rotated the handle with his right hand. In the first 4 to $5 \mathrm{sec}$ after he began the operation, the oxyHb rose. Throughout the course of the operation, the oxyHb rose and fell, but its value was always higher that that before the operation began. The oxyHb did not fall to the base level which existed before the operation until after completion of the operation. The results thus indicate that it is possible to determine the change in oxyHb in the motor cortex relating to handle rotation. As shown in Figure 4, moreover, increasing the handle torque resulted in a greater change in oxyHb. In summary, the oxyHb in the motor cortex generally increased in correspondence with the handle rotation, and the degree of increase was largely dependent on the force used to rotate the handle.

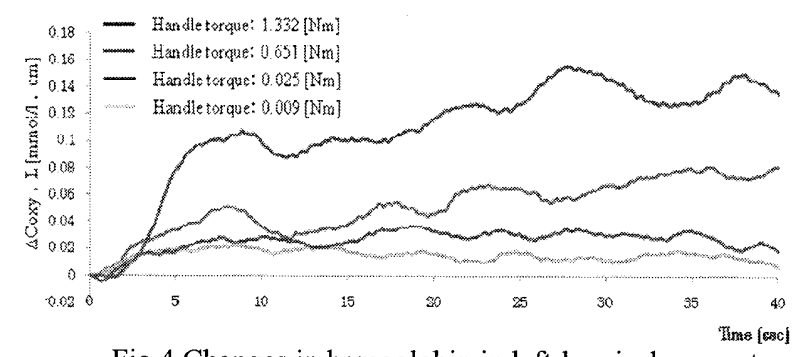

Fig.4 Changes in hemoglobin in left-hemisphere motor cortex during handle rotation with right hand

\section{Operation of general-purpose lathe}

In lathe working, which is a type of machining, a cylindrically shaped workpiece is fixed on the lathe and rotated, and a tool bit is pressed against the workpiece to machine it. Broadly classified, lathe working is performed either on NC lathes, in which the machining is performed automatically, or on general-purpose lathes, in which the machini $\mathrm{ng}$ is performed manually. The latter was used in the present study.

The general-purpose lathe contains a chuck for holding the workpiece, a toolpost which holds the tool bit, a carriage with a handle for longitudinal movement of the workpiece, a crosspiece with a handle for transverse movement of the toolpost, and a lever for actuation of the main spindle. The workflow essentially consists of: (1) attachment of the tool bit to the toolpost, (2) attachment of the workpiece to the chuck, (3) initiation of main spindle 
rotation, (4) alignment of the zero point, and (5) rotation of the handles to maintain tool bit engagement and thus cut the workpiece to the target configuration. Generally, the cutting speed (the rotational speed of the workpiece), the cutting depth (thus, the transverse position of the tool bit), and the feed rate (the rate of longitudinal movement by the tool bit) are controlled by the machinist in accordance with the workpiece material, its shape, the type of tool bit used, and the nature of the machining design. Together, these elements are referred to as the cutting conditions, and their control is the key factor determining lathe operating skill.

The above process steps (1) to (3) are preparatory steps. It is steps (4) and (5) that require a particularly high level of skill, and which are the subject of the present study. In this study, moreover, the recommended cutting conditions were set in accordance with the ideal cutting conditions based on the cutting target material and diameter.

\section{VR system for transmission of lathe operating skill}

In these trials, the operator stood in front of a screen, wearing a head tracking device enabling detection of the position and orientation of his head, and polarizing glasses. The operator thus had a stereoscopic view of the lathe image which was projected on the screen, and could manipulate the model handles which were located near his hands, as shown in Figure 5, and synchronized with the screen image. One handle was modeled on the carriage handle of an actual lathe for longitudinal movement, and the other on a toolpost handle for transverse movement. Each handle unit contained the handle, a magnetic brake, and an encoder. The handle and the magnetic brake were attached coaxially on the same crankshaft, and the encoder was linked to each crankshaft by coupling. The magnetic brakes imparted a sensation of a force on the operator when he rotated the handle. Simultaneously, a signal was sent from the encoder, the information was read by a PIC microcontroller, and the rotational speed was transmitted to a personal computer (PC). From the rotational speed, the number of rotations was determined and the depth of the cut was computed from the positional relationship of the tool bit and the workpiece. Based on this information, an image of the processing state was constructed and projected onto the screen. Figure 6 shows the projected screen image. Scale markings are present on actual lathe handles, but none were provided on the fabricated system handles and they were therefore shown in the image. As an added training function in the virtual system which simulated one aspect of OJT, it was possible to show not only a reproduction image of the actual object but also the feed rate in comparison with the feed rate of the recommended cutting conditions. During the operation, processing sounds were provided by an ITU-R5.1ch speaker system, to present the changes in sound when the tool bit came into contact with the workpiece and the various sounds depending on the cutting state. In this way, it was possible to present haptic, visual, and auditory information to the trainee.

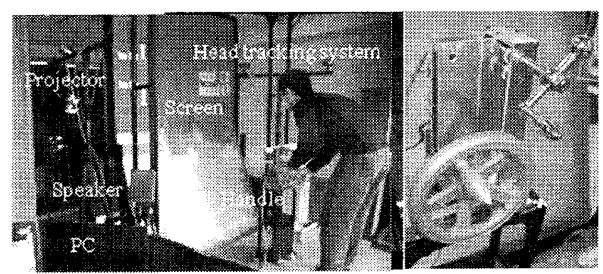

Fig.5 VR system for transmission of lathe operating skill

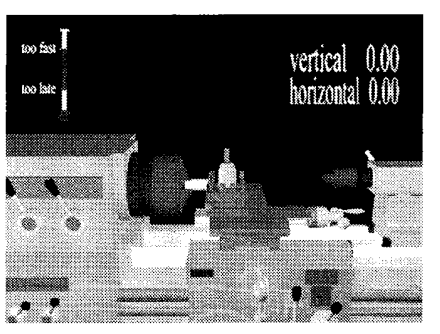

Fig.6 VR lathe 


\section{Brain activation response during lathe operation are measured by NIRS}

\subsection{Measurement of brain activation response by NIRS during lathe operation}

In these trials, measurements relating to the subject's brain activation level were performed during lathe operation in the VR lathe environment and in the actual lathe environment. The subject was a male in his twenties with experience in lathe operation. The trials in the VR environment were performed in alternating segments of observation of another person operating the lathe and operation by the subject himself. The parts of the brain measured by NIRS were the motor cortex and visual cortex in combination, and the frontal lobes and visual cortex in combination. For each combination, the trial was performed four times. The measurement positions are shown in Figure 7. In the actual lathe environment, similarly, the subject observed and performed the lathe operation by turns, each eight times. The workpiece material was aluminum. The cutting conditions, of which the subject was instructed in advance, were: cutting depth, $0.2 \mathrm{~mm}$; cutting length, $20 \mathrm{~mm}$. The time of the operation (the "task time") was $40 \mathrm{sec}$, and the non-operating time (the "rest time") was $80 \mathrm{sec}$.

\subsection{Analysis of brain activation response measurements by NIRS during lathe operation}

\subsubsection{Trials in the VR-lathe environment}

Figure 8 shows the changes in oxyHb levels in the motor cortex and visual cortex during the observation of operation in the VR environment by another person. As shown, the $\mathrm{oxyHb}$ in the visual cortex tended to decline in both the right and the left hemisphere. A decline in oxyHb in the motor cortex was also observed. To investigate whether the decline in oxyHb was attributable to the task observation, a test for a significant difference between oxyHb during the task time and during rest time was performed using the Holm-Bonferroni method. The level of significance was taken as $1 \%$. The results of this test showed a significant difference at almost all of the measurement positions in the visual cortex and motor cortex between the task time and the rest time, thus indicating that the measured decline in oxyHb was actually attributable to the task observation. In regard to the positions in which there was no significant difference, moreover, a test performed with the assumption that the task-time oxyHb increased showed no significant difference from the oxyHb change during the rest time. In summary, the results indicate that, in the case of observing another person perform the lathe operation in the VR lathe environment, the in the motor cortex and the visual cortex decline or do not change.

Figure 9 shows the results of the motor cortex and visual cortex measurements during operation of the VR lathe by the subject himself. In this case, oxyHb increased at almost all measurement positions in both the motor cortex and the visual cortex. In the visual sensory area, the oxyHb increase was larger in the upper positions than in the lower positions. Also, the increase in oxyHb was larger in the right hemisphere than in the left. In these trials, again, with a significance level of $1 \%$ as tested by the Holm-Bonferroni method, a significant difference was found between the task time and the rest time in almost all measured positions in the motor cortex and the visual cortex. It can therefore be concluded that the increase in oxyHb as measured by NIRS was attributable to the performance of the lathe operation. In the motor cortex, the positions in which the oxyHb increase was particularly large were in the vicinity of the temporal region which responds to hand movement and the parietal region which responds to trunk movement. In the visual cortex, the response at the lower measurement positions was particularly strong. The increase in oxyHb during performance of the operation was particularly large in comparison with the oxyHb changes during observation of the performance by another person. In the frontal cortex also, as shown in Figure 10, the oxyHb increase was clearly larger during operation 
by the subject than in observation of operation by another person.

Taken together, the results of the trials in the VR environment show that oxyHb tends to

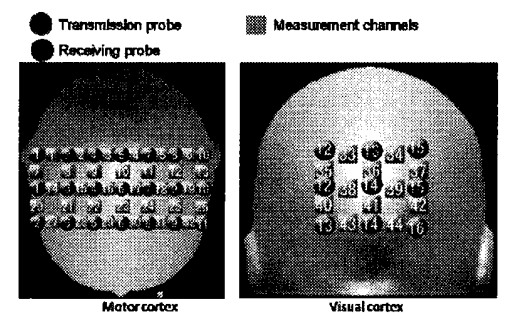

Fig.7 Measurement regions during lathe operation

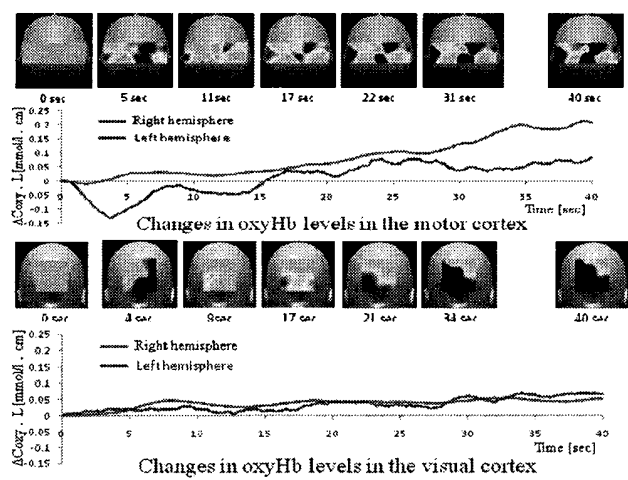

Fig.9 Brain activation response during performance of VR-lathe operation
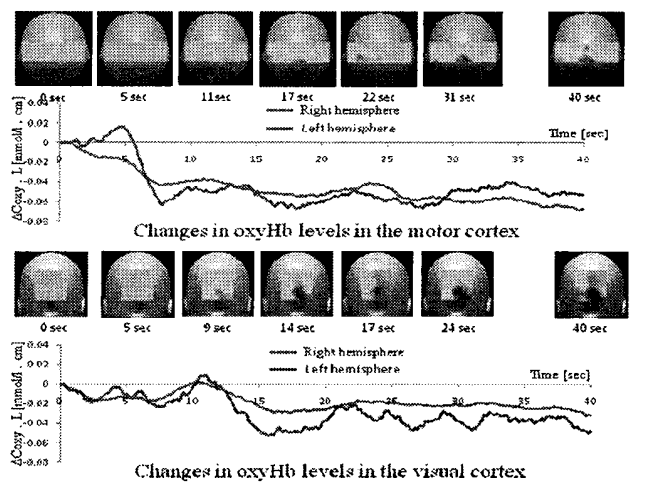

Fig.8 Brain activation response during observation of VR-lathe operation by other person

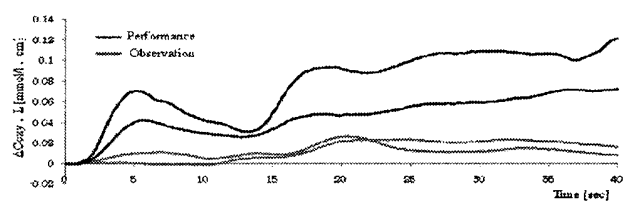

Fig.10 Brain activation responses in frontal lobe during observation and performance of VR-lathe operation

decline while watching someone else perform the lathe operation, but increases substantially while performing the operation. In short, they show that brain activity is greater while performing the lathe operation than while seeing it performed.

\subsubsection{Trials in the real-lathe environment}

Figure 11 shows the change in oxyHb level in the motor cortex and the visual cortex of the subject as he observed the operation of the actual lathe by another person. Although the change was small, in this case as in the case with the VR lathe, a decline in oxyHb was observed in the visual cortex and the motor cortex. The decline was again tested for significance by the Holm-Bonferroni method with a significance level of $1 \%$ to investigate whether it was attributable to the task, and the results again showed a significant difference between the task time and the rest time, for the measured positions in the overall visual cortex and nearly all of the motor cortex, thus indicating that the oxyHb decline was attributable to observation of the task. For the positions in which there was no significant difference, no significant difference from the change in hemoglobin during the rest time was found when it was assumed that the oxyHb increased during the task observation. In summary, the results indicate that, during observation of the lathe operation by another person in the real-lathe environment, oxyHb in the visual cortex and the motor cortex declined or did not change. 
Figure 12 shows the results for the motor cortex and the visual cortex during real-lathe operation by the subject. As before, to investigate whether the increase in oxyHb was attributable to the task, the results were tested by the Holm-Bonferroni method with a significance level of $1 \%$. The test results showed a significant difference between the rest time and the task time at nearly all measured positions in the motor cortex and the visual cortex, thus indicating that the increase in oxyHb was actually caused by the task performance. The measurement positions in the motor cortex that showed particularly large increases in oxyHb were in the temporal cortex. In the visual cortex, the oxyHb increase was particularly large in the lower positions. In the frontal cortex, as shown in Figure 13, the increase in oxyHb was larger during performance of the operation than during observation of its performance. The results thus indicate that in the real-lathe operation, as in the VR-lathe operation, the brain activation response was larger during performance of

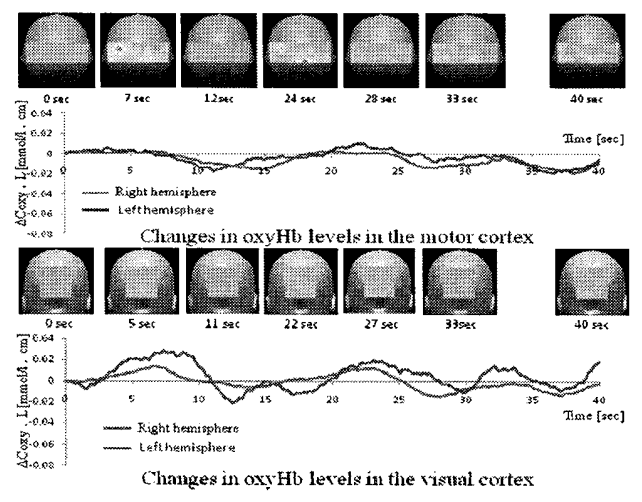

Fig. 11 Brain activation responses during observation of real-lathe operation

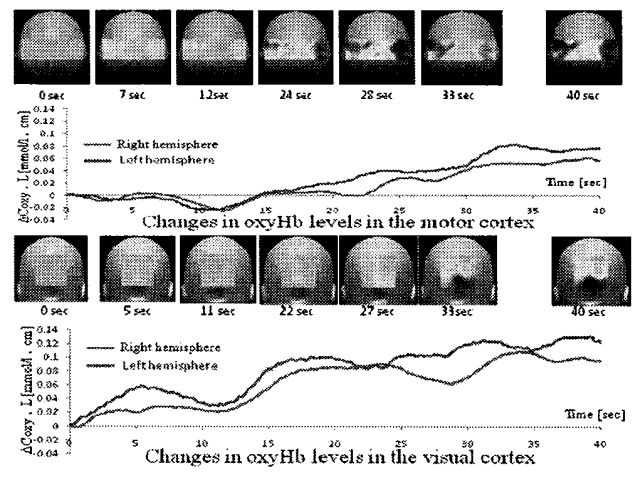

Fig.12 Brain activation responses during performance of real-lathe operation

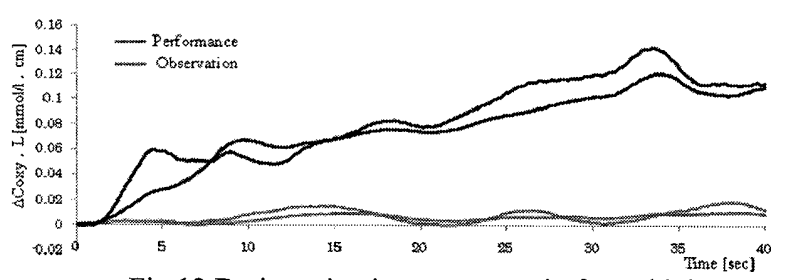

Fig.13 Brain activation responses in frontal lobe during observation and performance of real-lathe operation

the lathe operation than during observation of its performance by someone else.

\subsubsection{Brain activation response during performance of VR-lathe and real-lathe operation}

In the real-lathe operation by the subject, oxyHb was found to increase greatly in the lower measurement positions in the visual cortex. This may be attributed to the relative location of the workpiece and the tool bit on the actual lathe. As shown in Figure 14, both were below the usual line of sight of the subject, who therefore had to lower his gaze in their direction during the operation. During VR-lathe operation by the subject, similarly, the oxyHb increased greatly in the lower measurement positions of the visual cortex. As the VR lathe was presented in the same size and position as the actual lathe, it was similarly necessary for the subject to lower his gaze during the operation, thus presumably resulting in the large increase in oxyHb in those measurement positions in the lower portion of the visual cortex. 
In the VR-lathe operation by the subject, oxyHb increased greatly in the temporal region of the motor cortex. In the real-lathe operation, similarly, the increase in oxyHb was larger in the measurement positions in the temporal region of the motor cortex than in other regions. The temporal region of the motor cortex is the region of response to hand movement. In real-lathe operation, the movement of both hands is the primary movement, as they must be used to manipulate the handles. The same circumstances applied in the VR-lathe environment, and oxyHb was similarly found to increase greatly in the temporal region of the motor cortex. Figure 15 shows the oxyHb change in the motor cortex in the temporal region during the subject's performance of the VR-lathe and the real-lathe operation, and Figure 16 shows the oxyHb change in the frontal region. As shown, in both the real and the VR environments, oxyHb tended to increase as the operation proceeded, and the fluctuations also tended to increase. When the largest change in oxyHb was taken as 1 and all of the values were normalized, comparison of the results showed qualitative agreement in oxyHb changes in the motor cortex between the VR-lathe and real-lathe operation, but it also showed larger fluctuations in oxyHb change in the VR-lathe operation. Comparing the results for the right and left hemispheres, in the right hemisphere the oxyHb changes in the VR and real environments largely coincided but in the left hemisphere, although the overall trend of change during operation in the two environments showed qualitative agreement, the variation was larger in the VR environment. A difference between

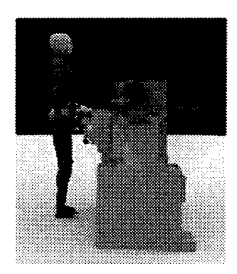

Line of tight

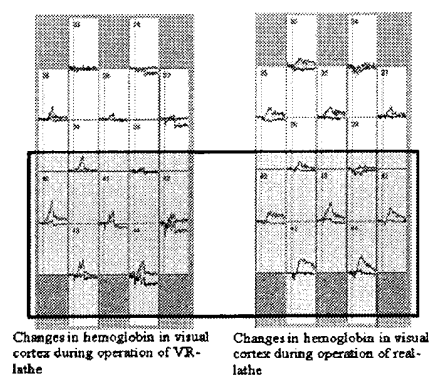

Fig. 14 Line of sight and change in Hemoglobin in visual cortex

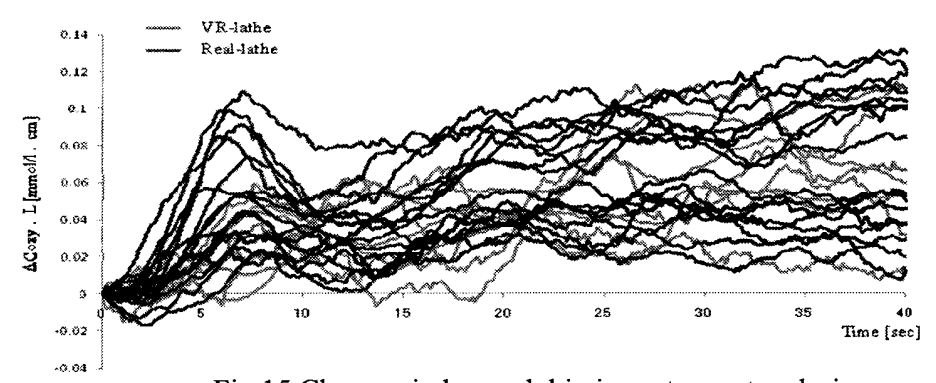

Fig.15 Changes in hemoglobin in motor cortex during Performance of VR- and real-lathe operation the two environments was also found in the oxyHb of the parietal region. In the VR environment, the oxyHb increased greatly in the vicinity of the parietal region, but in the real environment the increase was small. The parietal region of the motor cortex responds to trunk movement. As was seen from the experimental results of the trials for waist-bending and center of gravity movement, the change in oxyHb in the vicinity of the motor cortex of the parietal region depends in large part on these movements. This suggests that the difference between the two environments regarding oxyHb change in the parietal region was at least partly attributable to a difference in the posture of the subject during his performance of the operation.

In the frontal lobes, the oxyHb increase was larger during real-lathe operation by the subject than during VR-lathe operation. When the maximum oxyHb change was taken as 1 and all values were normalized, the results showed a qualitative agreement between the two modes of operation.

In summary, the above results show very close agreement between the trends in frontal 
cortex, motor cortex, and visual cortex activation responses during VR-lathe and real-lathe operation. This shows that essentially the same movements were performed by the operator with both lathes, and thus indicates that the lathe operation in the VR environment very closely mimics that in the real environment.

\section{Conclusion}

In this paper, brain activation responses at various NIRS measurement positions during the performance of three basic movements showed that the positions of change in oxyHb were clearly influenced by direction of movement and body posture at the time of the movements, and that the degree of change was largely governed by the level of force which was applied. The results also showed that the change in oxyHb was delayed by several seconds from the time of the actual movement. In the light of these findings, NIRS measurement was performed to investigate brain activation response during manual operation of a VR lathe and an actual lathe. The results showed qualitative agreement in the trends of oxyHb change for operation in the VR-lathe environment and the real-lathe environment. The results also showed that, in both environments, performance of the lathe operation induces greater changes in oxyHb than observation of its performance by another person, and thus that the brain is more highly activated by performance of the operation than by its observation.

The results, taken together, show that brain activation response during the performance of lathe operation in the VR environment is very close to that which occurs during operation in the actual environment, and thus indicate that training in such a VR environment will be effective and useful. In the future, we plan to perform more detailed analyses of movements performed during lathe operation and the relationship between the process of developing lathe operation skills and brain activation responses, and to apply the findings to more effective virtual learning.

\section{References}

(1) The Ministry of Health of Japan, Analysis of Labor Economy, (2005), pp.162-176.

(2) Kimura, Y., Taniguchi, Y., Fujinami, M., Factor to promote OJT in manufacturing, Journal of the Japan Institute for Labor policy and Training, No. 26, (2007)

(3) Watanuki, K., Knowledge Acquisition and Job Training for Fundamental Manufacturing Technologies and Skills by Using Immersive Virtual Environment, Journal of Japanese Society for Artificial Intelligence, Vol. 22, No. 4, (2007), pp.480-490. 